Editorial

\title{
Wearable Technology: A New Era of Gadgetry Innovation
}

\author{
Haider Khaleel \\ 1801 East Cotati Ave, Rohnert Park, California, USA
}

Today, wearable technology comes in a wide variety of forms, from wrist and arm bands to eyewear, footwear and smart textiles. The number of wearable electronic devices available in today's electronics market including smart watches, activity trackers, helmets and smart clothing seems to grow substantially. In fact, this technology is recognized as one of the hottest trends and one of fastest growing technologies in the modern world. According to a recent market study, the international revenue of this technology is estimated to be 45 billion USD in 2016 and over 300 billion USD in 2028 (Hu, 2010).

Wearable electronics, which have the merits of portability, mechanical flexibility and potentially, reconfigurability, would significantly broaden the applications of modern devices and gadgets. This technology is in fact being established as an appealing alternative to the conventional hand-held electronics technology which is based on rigid platforms. It is worth mentioning that recent advancements in the development of miniaturized and efficient energy storage and self-powered electronic components have facilitated the commercial success of this technology (Khaleel et al., 2012).

One can already observe a growing demand for commercial wearable products in today's electronics market (think Google Glass, Fitbit and Apple Watch). There is an immense variety of smart watches, health monitors, fitness bands, smart clothing and other wearable electronics on the shelves, which clearly indicates that the wearable technology is on the edge of becoming mainstream. Moreover, in light of the social media revolution, wearable technologies could serve as an excellent platform for modern consumers who tend to capture and share the progress and activity of their lifestyles digitally.

Generally speaking, wearable technologies aim at integrating technology into everyday life activities to maximize the quality and convenience of consumers' life. For example, wearable activity and health trackers that monitor the vital signs of a user, such as heart rate, glucose level, temperature, blood pressure, burned calories and sometimes even sleep and stress patterns are very popular nowadays (Nathan et al.,
2012). It is also expected that in the near future, more types of biometric data would be captured via additional sensors embedded within the wearable device and naturally, it is anticipated to see personalized apps that link these advanced functions to smart phones, tablets and the internet cloud.

Tracking, monitoring and identifying people and objects by means of low-power and low-cost Radio Frequency Identification (RFID) technology is another great example of wearable technology. This technology has already flourished due to the advances in material science and power efficient electronic components manufacturing (Lakafosis et al., 2010).

An ultimate example of this technology would be the Google Glass which is probably the most publicized wearable product. Google Inc. is also recognized as one of the most adopting corporations of this technology. The Glass is basically a pair of glasses equipped with a built- in microprocessor and a bunch of essential peripherals such as a camera, display, finger-pad and a microphone. Via these peripherals a user can access endless streams of information and capture instant activities through a friendly, easy to use interface. Furthermore it utilizes voice recognition technology to type messages or perform commands. Google Glass accesses information on the internet via two wireless technologies: Wi-Fi and Bluetooth which are hosted by the wireless service of the user's cell phone.

Obviously, the number of applications and fields that could hugely benefit from this technology is virtually unlimited. Some of the fascinating applications that utilize the Glass are in the fields of personal communication, medicine, entertainment, sport, tourism and security. Google is also working on optimizing a smart contact lens with an embedded miniaturized sensor that measures glucose levels in tears and forward the data to a smart phone through a compact wireless transmitter which provides glucose information for diabetic patients (Novartis, 2014).

On the downside, there are a couple of noteworthy technical challenges that currently form an obstacle in the way of further deployment and extended functionality of this technology. The first is an obvious one: The limitations of the current battery technology in terms of efficiency, size and sustainability. Secondly, for 
most applications, wearable devices require their integrated components to have the merits of flexibility and mechanical robustness simultaneously; i.e.: They must tolerate high levels of bending, flexing and rolling repeatability. Additionally, weather and environmental factors (humidity, heat, pressure, etc.) have negative effects on the performance of wearable devices which need to be addressed and analysed ( Raad et al., 2013). This consequently raises a trade-off between convenience and product durability. However, solutions to these aspects will eventually improve over time, allowing manufacturers to optimize devices further. Advancement in nanotechnology and printed circuits seems to be steering in the right direction when it comes to the fields of sustainable energy and circuit miniaturization. Additionally, with the extremely fast emergence of new technological trends, consumers tend to take time to fully embrace and absorb such technologies. In fact, market analysts anticipate that as with laptops and tablets, wearable technology will spend five to ten years of its age targeting vertical markets before becoming mainstream triggered by the emergence of a breakthrough product.

Obviously, the applications of wearable technologies are extremely powerful and virtually countless and would potentially revolutionize our lives on various levels. Therefore, all aspects of this vital topic are attracting serious attention from government, academia and R\&D firms aiming at enforcing a faster technology adoptability.

\section{References}

$\mathrm{Hu}$, J., 2010. Overview of flexible electronics from ITRI's viewpoint. Proceedings of the 28th VLSI Test Symposium, Apr, 19-22, IEEE Xplore Press, Santa Cruz, CA., pp: 19-22.

DOI: 10.1109/VTS.2010.5469608

Khaleel, H.R. H.M. Al-Rizzo, D.G. Rucker and S. Mohan, 2012. A compact polyimide-based UWB antenna for flexible electronics. IEEE Lett. Antennas Wireless Propagat., 11: 564-567. DOI: 10.1109/LAWP.2012.2199956

Lakafosis, V., A. Rida, R. Vyas, L. Yang and S. Nikolaou et al., 2010. Progress towards the first wireless sensor networks consisting of inkjetprinted, paper-based RFID-enabled sensor tags. Proc. IEEE, 98: 1601-1609.

DOI: 10.1109/JPROC.2010.2049622

Nathan, A., A. Ahnood, M.T. Cole, S. Lee and Y. Suzuki et al., 2012. Flexible electronics: The next ubiquitous platform. Proc. IEEE, 100: 1486-1517. DOI: 10.1109/JPROC.2012.2190168

Novartis, 2014, Novartis to license Google "smart lens" technology. Novartis.

Raad, H.R., A.I. Abbosh, H.M. Al-Rizzo and D.G. Rucker, 2013. Flexible and Compact AMC Based Antenna for Telemedicine Applications. IEEE Trans. Antennas Propagat., 61: 524-531. DOI: $10.1109 /$ TAP.2012.2223449 